\title{
Alternative splicing at NAGNAG acceptors in Arabidopsis thaliana SR and SR-related protein-coding genes Stefanie Schindler*1, Karol Szafranski ${ }^{1}$, Michael Hiller ${ }^{2}$, Gul Shad Ali³, Saiprasad G Palusa ${ }^{3}$, Rolf Backofen ${ }^{2}$, Matthias Platzer ${ }^{1}$ and Anireddy SN Reddy ${ }^{3}$
}

\author{
Address: ${ }^{1}$ Genome Analysis, Leibniz Institute for Age Research - Fritz Lipmann Institute, Beutenbergstr. 11, 07745 Jena, Germany, ${ }^{2}$ Institute of \\ Computer Science, Bioinformatics Group, Albert-Ludwigs-University Freiburg, Georges-Koehler-Allee 106, 79110 Freiburg, Germany and \\ ${ }^{3}$ Department of Biology and Program in Molecular Plant Biology, Colorado State University, Fort Collins, CO, USA \\ Email: Stefanie Schindler* - sschindl@fli-leibniz.de; Karol Szafranski - szafrans@fli-leibniz.de; Michael Hiller - hiller@informatik.uni- \\ freiburg.de; Gul Shad Ali - gsali@lamar.colostate.edu; Saiprasad G Palusa - saigoud@lamar.colostate.edu; \\ Rolf Backofen - backofen@informatik.uni-freiburg.de; Matthias Platzer - mplatzer@fli-leibniz.de; Anireddy SN Reddy - reddy@colostate.edu \\ * Corresponding author
}

Published: 10 April 2008

BMC Genomics 2008, 9:159 doi:10.1186/147|-2164-9-159
Received: 20 September 2007

Accepted: 10 April 2008

This article is available from: http://www.biomedcentral.com/I47I-2164/9/159

(c) 2008 Schindler et al; licensee BioMed Central Ltd.

This is an Open Access article distributed under the terms of the Creative Commons Attribution License (http://creativecommons.org/licenses/by/2.0), which permits unrestricted use, distribution, and reproduction in any medium, provided the original work is properly cited.

\begin{abstract}
Background: Several recent studies indicate that alternative splicing in Arabidopsis and other plants is a common mechanism for post-transcriptional modulation of gene expression. However, few analyses have been done so far to elucidate the functional relevance of alternative splicing in higher plants. Representing a frequent and universal subtle alternative splicing event among eukaryotes, alternative splicing at NAGNAG acceptors contributes to transcriptome diversity and therefore, proteome plasticity. Alternatively spliced NAGNAG acceptors are overrepresented in genes coding for proteins with RNA-recognition motifs (RRMs). As SR proteins, a family of RRMcontaining important splicing factors, are known to be extensively alternatively spliced in Arabidopsis, we analyzed alternative splicing at NAGNAG acceptors in SR and SR-related genes.
\end{abstract}

Results: In a comprehensive analysis of the Arabidopsis thaliana genome, we identified 6,772 introns that exhibit a NAGNAG acceptor motif. Alternative splicing at these acceptors was assessed using available EST data, complemented by a sequence-based prediction method. Of the 36 identified introns within 30 SR and SR-related protein-coding genes that have a NAGNAG acceptor, we selected 15 candidates for an experimental analysis of alternative splicing under several conditions. We provide experimental evidence for 8 of these candidates being alternatively spliced. Quantifying the ratio of NAGNAG-derived splice variants under several conditions, we found organ-specific splicing ratios in adult plants and changes in seedlings of different ages. Splicing ratio changes were observed in response to heat shock and most strikingly, cold shock. Interestingly, the patterns of differential splicing ratios are similar for all analyzed genes.

Conclusion: NAGNAG acceptors frequently occur in the Arabidopsis genome and are particularly prevalent in SR and SR-related protein-coding genes. A lack of extensive EST coverage can be compensated by using the proposed sequence-based method to predict alternative splicing at these acceptors. Our findings indicate that the differential effects on NAGNAG alternative splicing in SR and SR-related genes are organ- and condition-specific rather than gene-specific. 


\section{Background}

Alternative splicing is an important mechanism for regulating gene expression at the post-transcriptional level and contributes to proteome complexity [1-3]. This widespread process comprises various mechanisms such as exon skipping, mutually exclusive exons, intron retention, or the usage of alternative 5' or 3' splice sites [4]. Alternative splicing has been extensively studied in mammals but less in plants. Recent evidence indicates more than $60 \%$ of the genes in the human genome alternatively spliced [5] compared to about $20-30 \%$ in plants $[6,7]$, based on EST/ cDNA data. A hallmark of plant introns is their relatively short length ( 150 vs. 740 nt in humans, on average) [8] and Uridine-richness [9]. Furthermore, plant introns exhibit a weaker polypyrimidine tract than mammals $[2,9]$. Datasets of spliced alignments from the TIGR $[6,10]$ and RIKEN [11] databases of full-length cDNAs and ESTs provide useful annotated versions of the Arabidopsis genome sequence for the detection of various alternative splice events. Based on the final TIGR annotation release, a total of 26,207 genes are annotated in Arabidopsis [12]. Although splicing machinery is generally conserved between plants and animals [2,9], plants exhibit a much higher fraction of retained introns (more than $40 \%$ of the events) compared to $\sim 10 \%$ reported for humans [5-8].

The fidelity of intron excision from a pre-mRNA relies on the precise recognition of exonic and intronic sequence signals and the complex interplay of different spliceosomal RNAs and proteins. Among these, SR proteins direct splice site selection by recognizing splice sites and splicing regulatory sequences (enhancers and silencers), thereby facilitating spliceosome assembly $[13,14]$. SR proteins are important factors for constitutive and alternative splicing. This evolutionary conserved protein family contains structurally related proteins that possess one or two RNA-recognition motifs (RRM) at the N-terminus and a C-terminal arginine/serine-rich (RS) domain [15,16]. A recent genome-wide survey on Arabidopsis splicingrelated genes revealed variations in SR proteins and hnRNP proteins between plants and mammals, suggesting plant-specific differences in splicing-regulation mechanisms [17]. The A. thaliana genome encodes 19 SR proteins, almost twice as many as in humans $[18,19]$. They can be subdivided into seven families [20]. Whereas SF2/ASF, 9G8 and SC35 are orthologues between plants and metazoa, the RS, RS2Z, SCL and SR45 subfamilies seem to be plant-specific. Most of the SR protein genes are themselves alternatively spliced to a great extent in Arabidopsis [20,21]. Fifteen of the 19 genes coding for SR proteins in Arabidopsis undergo alternative splicing and produce at least 95 transcripts [21]. In some cases, it was shown that alternative splicing correlates with the intron length [22]. Splicing patterns of Arabidopsis SR protein genes are under tight spatio-temporal control, leading to a different abundance of splice variants in different tissues and at developmental stages [21,23-26]. Several plant SR proteins have been shown to regulate the splicing of their own transcripts and transcripts of other SR genes [25,2729]. Environmental conditions can also modulate the splicing pattern of a gene, as shown by the temperature dependent alternative acceptor selection of SR1B/SR1 in Arabidopsis [30]. Furthermore, stresses such as exposure to cold, heavy metals or anaerobiosis, affect the efficiency or patterns of splicing [21,31-33], but the mechanisms by which some types of stress influence alternative splicing in plants are largely unknown.

In plants and mammals, the most frequent distance between alternative acceptors is $3 \mathrm{nt}[6,34]$. Such tandem acceptors have been termed NAGNAG acceptors based on the existence of a NAGNAG acceptor motif $(\mathrm{N}=\mathrm{A}, \mathrm{C}, \mathrm{G}$, T) $[35,36]$. In the NAGNAG motif, the upstream acceptor is termed the E-acceptor (since the downstream NAG becomes exonic upon splicing at this site) and the downstream one the I-acceptor (since the whole tandem becomes intronic) [36]. Alternative splicing at NAGNAG acceptors is widespread in many species $[37,38]$ and also in plants [6,39] with Caenorhabditis elegans [36] being the only exception known so far. The selection of either AG in the splicing process results in the insertion/deletion (indel) of the I-acceptor NAG in mRNAs. This leads to diverse effects at the protein level with the majority of the events involving the indel of a single amino acid. A fraction of these events is estimated to be under purifying selection, suggesting an evolutionary conserved function [40]. Interestingly, it was demonstrated that the distribution of NAGNAG acceptors is highly similar between mammals and plants, for example, polar amino acid residues were found to be predominantly affected in both kingdoms [36,41].

An example for functional alternative splicing in Arabidopsis is a TAGCAG acceptor affecting the RNA-binding domain of the U11-35K protein that results in different binding affinity for SR proteins and the U11 snRNA in vitro [42]. In contrast, both splice variants derived from a CAGCAG acceptor in the tomato prosystemin gene are active signaling components of the wound response pathway, without detectable functional differences [39].

Previously, we found that human genes coding for RNA binding proteins including many splicing factors are preferentially equipped with NAGNAG acceptors [36]. Here, we observed a similar overrepresentation of NAGNAG acceptor motifs in Arabidopsis. This agrees with a very recent study, where NAGNAG alternative splicing was also found to be accumulated in genes for RNA-binding proteins in Arabidopsis [41]. 
Since splice variants of splicing factors may have consequences for alternative splicing and its regulation [43], we investigated alternative NAGNAG splicing at SR and SRrelated genes. We determined the splicing ratios of 15 NAGNAG acceptors in splicing factors for several plant tissues, seedlings of different ages and in response to cold and heat stresses. We detected organ-specific variations and differences between the developmental stages. Cold stress was found to induce the most remarkable changes in the splicing ratios.

\section{Results \\ NAGNAG acceptors are frequent in the Arabidopsis genome}

A comprehensive list of introns was constructed from the annotated Arabidopsis genome sequence based on RIKEN [11] and TIGR [6,10] cDNA sequences. Out of 112,934 intron-exon boundaries (taken from 26,207 annotated protein-coding genes), 6,772 showed a NAGNAG motif within 5,381 genes (Additional table 1). Thus, 6\% of all introns and $21 \%$ of all annotated genes in Arabidopsis harbor a genomic NAGNAG acceptor motif. For comparison, in human, 5\% of introns and 30\% of genes harbor such a motif [36]. We categorized all Arabidopsis cases according to their EST coverage (Additional table 2). In 229 cases (3\%), no EST support exists for either of the possible acceptor sites. In 1,899 cases (28\%), a single EST supports either acceptor. Out of the remaining 4,644 cases with minimally required EST coverage (two or higher, 69\%), 242 cases (5\%) have supporting ESTs for both acceptor sites. Naturally, EST-based evidence for alternatively spliced NAGNAGs depends on their isoform frequencies and the EST coverage, which is low in Arabidopsis compared to other species such as human or mouse. For example, if a minor isoform occurs with $10 \%$ frequency, at least 29 ESTs are necessary to reach a probability of $95 \%$ that it will be found (binomial test). Hence, in many cases, native alternative splicing remains undetected, and certainly more NAGNAG sites than those indicated by the current transcript data are expected to be alternatively spliced.

In order to overcome this limitation of EST coverage we established a sequence-based prediction method for alternative splicing at NAGNAG acceptors. There is evidence that a narrow context of flanking nucleotides captures most of the information relevant for prediction of the splice variant ratio $[44,45]$. Conservatively, we chose a heptameric context NAGNAGN, comprising the two acceptor AG dinucleotides and three additional variable positions, and divided all NAGNAG cases into 64 heptamer classes. The EST counts within each of the classes were pooled, and the resulting splicing variant ratio (fraction of E-transcripts) was considered representative for all cases of that heptamer class. For example, the average fre- quency of the E-isoforms of 55 observed CAGCAGA acceptors, based on 227 pooled ESTs, is $48 \%$, and this was taken as the predicted frequency for any CAGCAGA acceptor motif (Additional Table 3). The validity of the heptamer-based approach is corroborated by the finding that maximum-likelihood estimators mostly agree between models for Arabidopsis and human. The high level of agreement is explained by the basic finding that the splicing ratios follow the basic rules of sequence preferences seen for isolated 3' splice sites: position -3 with $\mathrm{C} \geq \mathrm{T}>\mathrm{A}$ $>\mathrm{G}$, position +1 with $\mathrm{G} \geq \mathrm{A}>\mathrm{T}>\mathrm{C}$ (data not shown).

Applying this method to the 2,128 cases with insufficient EST coverage (less than two ESTs), 482 (23\%) are predicted to have a minor transcript frequency of at least $10 \%$. Using this conservative threshold for isoform abundance gives a lower-bound estimate of the fraction of alternatively spliced NAGNAG sites. Applying this prediction method to all NAGNAG cases in Arabidopsis, 14\% are predicted to be alternatively spliced with a minor transcript frequency of at least $10 \%, 21 \%$ with $\geq 5 \%$, and $33 \%$ with $\geq 2 \%$, respectively. Interestingly, as EST coverage increases, NAGNAG acceptors are less often predicted to be alternatively spliced (<2 ESTs: 23\%, 2-5 ESTs: 11\%, >5 ESTs: $8 \%$ ). These results indicate that the occurrence of alternatively spliced NAGNAG acceptors is negatively correlated with the transcript levels of the genes.

\section{Many $S R$ and SR-related protein transcripts contain NAGNAG acceptors}

For identification of SR and SR-related genes we searched for characteristic protein signatures in the gene products associated with NAGNAG acceptors [46]. Of all Arabidopsis proteins, 84 proteins had RRM domains and are rich for R/S dipeptides. Of these 84, 19 were previously identified as SR proteins $[18,19]$, leaving 65 SR-related proteins. The intersection with NAGNAG cases gave 36 introns in 30 genes (Table 1). Thus, 36\% of SR and SR-related protein-coding genes exhibit NAGNAG acceptors ( 7 out of 19 SR, 23 out of 65 SR-related). This is significantly higher than the average frequency of NAGNAG-containing genes $(21 \%)$, even if we account for a higher fraction of multiexon genes and a slightly higher number of introns in the SR/SR-related gene family $(P=0.068$, permutation test). This finding is very similar to human where alternatively spliced NAGNAG motifs were found to be enriched in RRM-containing proteins [36].

SR33/SCL33 is the only case which exhibits EST support for alternative NAGNAG splicing (Table 1). Intriguingly, in 14 cases, the sequence-based prediction argues for the usage of both acceptor sites with a predicted minor transcript frequency of $2 \%$. This permissive $2 \%$ threshold was applied in narrowing the list of experimental candidates in order to retain those which have a substantial chance to 
Table I: NAGNAG acceptors in Arabidopsis SR and SR-related protein-coding genes. In summary, 36 NAGNAG-containing introns occur in $\mathbf{3 0}$ genes. Genes are classified into SR and SR-related protein coding genes. Splicing ratios are given as absolute EST counts ("\#"). Column 'heptamer motif' specifies the heptamer sequence of the NAGNAG acceptor sites used for the sequence-based prediction; here, "|" marks the annotated acceptor. Predicted E-transcript proportions are listed in column 'E-transcript predicted'. Gene names are grey shaded if they contain two NAGNAG acceptors.

\begin{tabular}{|c|c|c|c|c|c|c|c|c|}
\hline Gene & Name & SR & SR-related & Intron & $\begin{array}{l}\text { \# ESTs E- } \\
\text { transcript }\end{array}$ & $\begin{array}{l}\text { \# ESTs I- } \\
\text { transcript }\end{array}$ & Heptamer motif & $\begin{array}{c}\text { E-transcript } \\
\text { predicted (\%) }\end{array}$ \\
\hline At5g52040 & RS4I & $x$ & & 2 & 5 & 17 & AAG|CAG,G & 7 \\
\hline At4g31580 & RSZ22 & $x$ & & 3 & 15 & 0 & TAG|GAG,C & 99 \\
\hline At3gI3570 & SCL30a & $x$ & & 3 & 13 & 0 & TAG|GAG,G & 99 \\
\hline Atlg55310 & SR33/SCL33 & $x$ & & 3 & 0 & 8 & CAG,CAG $\mid \mathrm{A}$ & 48 \\
\hline Atlg 16610 & SR45 & $x$ & & 7 & 0 & 12 & CAG,CAGIG & 48 \\
\hline At $\lg 16610$ & SR45 & $x$ & & 9 & 0 & 12 & AAG,CAG|G & 7 \\
\hline Atlg 23860 & SRZ2I & $x$ & & 3 & 13 & 0 & CAG|GAG,A & 100 \\
\hline At2g24590 & SRZ22a & $x$ & & 3 & 8 & 0 & CAG|AAG,A & 98 \\
\hline Atlg07350 & & & $x$ & 2 & 21 & 0 & TAG|GAG,A & 100 \\
\hline Atlg22910 & & & $x$ & I & 7 & 0 & TAG|GAG,C & 99 \\
\hline Atlg53650 & CID8 & & $x$ & 4 & 7 & 0 & CAG|AAG,G & 97 \\
\hline Atlg60000 & cp29 & & $x$ & 1 & 6 & 0 & CAG|GAG,T & 100 \\
\hline Atlg60900 & U2AF65 & & $x$ & 2 & 3 & 0 & CAG|GAG,A & 100 \\
\hline Atlg60900 & U2AF65 & & $x$ & 4 & 0 & 2 & TAG,CAG|G & 17 \\
\hline Atlg76940 & & & $x$ & I & 2 & 0 & TAG|AAG,G & 83 \\
\hline At2g24350 & & & $x$ & 4 & 0 & 2 & AAG,CAG|T & 7 \\
\hline At2g24350 & & & $x$ & 1 & 5 & 0 & CAG|GAG,T & 100 \\
\hline At2g35410 & cp33 & & $x$ & 3 & 16 & 0 & TAG|GAG,T & 100 \\
\hline At2g37220 & cp29 & & $x$ & 2 & 22 & 0 & TAG|GAG,T & 100 \\
\hline At2g43370 & UII-35K & & $x$ & 4 & 0 & 6 & TAG,CAG|G & 17 \\
\hline At2g43370 & UII-35K & & $x$ & 5 & 5 & 0 & CAG|GAG,T & 100 \\
\hline At3g23830 & GR-RBP4/GRP4 & & $x$ & 3 & 15 & 0 & CAG|GAG,T & 100 \\
\hline At3g26420 & ATRZ-IA & & $x$ & 3 & 5 & 0 & TAG|AAG,G & 83 \\
\hline At3g51950 & & & $x$ & 4 & 6 & 0 & CAG|GAG,G & 100 \\
\hline At3g54230 & & & $x$ & 5 & 0 & 3 & GAG,CAG|G & 1 \\
\hline At3g54230 & & & $x$ & 6 & 0 & 3 & TAG,CAG|C & 25 \\
\hline At4g35785 & & & $x$ & 4 & 0 & 0 & CAG,AAG|T & 98 \\
\hline At4g36960 & & & $x$ & 8 & 5 & 0 & TAG|GAG,A & 100 \\
\hline At5g02530 & & & $x$ & 2 & 6 & 0 & TAG|GAG,A & 100 \\
\hline At5g03580 & PABP & & $x$ & I & 0 & I & GAG,TAG|G & 0 \\
\hline At5g09880 & & & $x$ & 1 & 5 & 0 & CAG|AAG,A & 98 \\
\hline At5g44200 & CBP2O & & $x$ & I & 4 & 0 & CAG|GAG,A & 100 \\
\hline At5g44200 & CBP2O & & $x$ & 7 & 6 & 0 & CAG|GAG,G & 100 \\
\hline At5g47320 & RPS 19 & & $x$ & 3 & 8 & 0 & TAG|GAG,T & 100 \\
\hline At5g53 180 & PTB & & $x$ & 7 & 0 & 7 & GAG,TAG|G & 0 \\
\hline At5g59950 & & & $x$ & I & 0 & 11 & TAG,CAG|A & 7 \\
\hline
\end{tabular}

be alternatively spliced. We selected 15 SR and SR-related protein-coding genes for experimental analysis, including SR33/SCL as a positive control (Table 2).

\section{Experimental evidence for NAGNAG isoforms in SR and $S R$-related protein genes}

For experimental detection of splice variants, cDNA from different adult plant organs (root, leaf, stem, inflorescence) and from callus and seedlings of different ages ( $3 \mathrm{~d}$, $5 \mathrm{~d}, 10 \mathrm{~d}, 15 \mathrm{~d}$ ) was sampled to cover a broad spectrum of transcript sources. Three independent RT-PCRs were performed per cDNA sample and gene, and splice variants were separated by capillary electrophoresis and subsequently quantified based on fluorescence intensity. We considered a NAGNAG candidate as alternatively spliced if the measurements indicated an average minor transcript frequency of at least two times the standard deviation. In a conservative approach, we evaluated the averages of the plant samples, in order to avoid extreme values from single samples that could cause false positives.

Eight cases of SR and SR-related protein-coding genes were found to be alternatively spliced at their NAGNAG acceptor sites (53\% of 15 tested, 22\% of NAGNAGs in this family; Table 2, Additional Table 4). In addition, the alternative splicing patterns of RS41 and SR33 were independently confirmed by Sanger sequencing of at least 100 clones (data not shown). Using the same approach, alter- 
Table 2: Experimental candidates with corresponding Etranscript proportions based on EST and experimental data. EST ratios are given as absolute EST counts. Column 'predicted' lists the E-transcript proportions obtained from the sequencebased prediction. Grey shaded values mark the cases where NAGNAG alternative splicing was validated (avg [minor isoform in organs, seedlings] $>2 \times$ avg [error]). At4g35785 is lacking EST data. Therefore, an EST-based E-transcript frequency cannot be shown and is indicated by '-'.

\begin{tabular}{lccc}
\hline \multicolumn{3}{c}{ E-transcript (\%) } \\
\hline Gene & EST & Predicted & Experiments \\
\hline RS4I & 23 & 7 & $16.7 \pm 0.6$ \\
SR33/SCL33 & 0 & 48 & $29.9 \pm 0.7$ \\
SR45i7 & 0 & 48 & $0.6 \pm 0.5$ \\
SR45i9 & 0 & 7 & $0.4 \pm 0.6$ \\
SRZ22a & 100 & 98 & $99.5 \pm 0.6$ \\
CID8 & 100 & 97 & $99.1 \pm 0.5$ \\
AtIg76940 & 100 & 81 & $98.8 \pm 0.9$ \\
At2g24350 & 0 & 7 & $3.2 \pm 1.5$ \\
ATRZ-IA & 100 & 83 & $98.9 \pm 1.4$ \\
At3g54230 & 0 & 25 & $2.3 \pm 0.5$ \\
At4g35785 & $-*$ & 98 & $97.4 \pm 0.3$ \\
At5g09880 & 100 & 98 & $99.0 \pm 0.7$ \\
At5g59950 & 0 & 7 & $2.8 \pm 0.4$ \\
UII-35K & 0 & 17 & $8.5 \pm 0.9$ \\
U2AF65 & 0 & 17 & $5.8 \pm 0.6$ \\
\hline
\end{tabular}

native NAGNAG splicing could not be detected in SR45i9 and SRZ22a, consistent with the quantitative capillary electrophoresis results. It is noteworthy that the relatively high frequency of alternative splicing in SR33/SCL33 was not indicated by the eight ESTs that exist for that transcript region.

In the tested cases, the E-acceptor was found to represent the minor acceptor in nearly all cases, and the prediction for alternative splicing was more often accurate for the major-I subclass compared to major-E subclass. This is consistent with the global case distribution evident from EST data, which divides into $13 \%$ constitutive I, $17 \%$ alternative major-I, $13 \%$ alternative major-E, $57 \%$ constitutive $\mathrm{E}$ cases (based on the 2\%-abundance threshold).

Genome-wide, the sequence-based prediction method suggested that $33 \%$ of NAGNAGs are alternatively spliced, producing minor isoforms with at least $2 \%$ frequency. For the 15 SR/SR-related cases that fulfill this prediction criterion, $53 \%$ were actually validated by our experiments. The prediction accuracy is positively correlated with the predicted minor transcript frequency. For example, cases predicted to have $2-5 \%$ minor transcripts are validated with a rate of $25 \%$ whereas cases predicted to have more than $5 \%$ minor transcripts are validated with a rate of $64 \%$ (Table 2). Consequently, a threshold of $2 \%$ seems to fully capture the fraction of likely alternatively spliced NAG-
NAG acceptors, as was intended for the selection of experimental candidates. Unfortunately, independent measures for the fraction of non-SR protein genes that undergo alternative NAGNAG splicing do not exist. However, based on the prediction results, we expect that SR/ SR-related protein genes have a slightly higher propensity for alternative splicing ( $42 \%$ versus 33\%).

\section{Organ-specific alternative splicing of NAGNAG acceptors and differential splicing ratios during development}

Splicing patterns of Arabidopsis SR protein genes are under tight spatio-temporal control, leading to a different abundance of splice variants in different tissues and at developmental stages [21,23-26]. Thus, we considered the occurrence of possible differences in the splice variant distribution in various plant organs (root, leaf, stem and inflorescence) and in callus. Based on the prior results, we similarly tested the cDNAs from those candidates, where the NAGNAG alternative splicing was successfully validated. In four cases (Figure 1, Table 3), a significant organspecificity was observed (ANOVA, Table 3). Interestingly, inflorescence tissue shows reduced splicing of the minor acceptor (mostly E-acceptor) in nearly all cases. This trend is also observed for the NAGNAG cases that do not show significant organ-specific splicing.

Next, we asked if the splicing ratios at the NAGNAG acceptors exhibit developmental variations. To this end, we analyzed cDNA derived from seedlings at the ages of $3 \mathrm{~d}$, 5d, 10d and 15d (Table 4, Figure 1). In four analyzed cases, statistically significant splicing ratio changes could be detected (ANOVA, Table 4). Comparing the values of the $3 \mathrm{~d}, 5 \mathrm{~d}, 10 \mathrm{~d}$ and $15 \mathrm{~d}$ probes, our results show a general trend towards increased minor acceptor usage with seedling development.

\section{NAGNAG splicing ratios under heat and cold shock}

Finally, we examined whether temperature stresses can modulate the NAGNAG splicing pattern, as was previously illustrated by temperature-controlled splicing ratios of SRp34/SR1 and other SR transcripts [21,30]. Seedlings were kept in hot or cold conditions and compared to an untreated control. Rather slight splicing ratio changes could be observed in the heat-shocked probes (Figure 1, Table 5). However, this difference was statistically significant in four cases (ANOVA, Table 5). In contrast, more obvious splicing ratio changes could be detected under cold shock (Figure 1). In six cases, a significant rise in minor acceptor usage was observed (ANOVA, Table 5), that clearly increased with the duration of treatment.

\section{Discussion}

SR proteins are important regulatory splicing factors and facilitate the correct interplay of components of the splicing machinery. Alternative splicing of SR protein genes is 
SR33

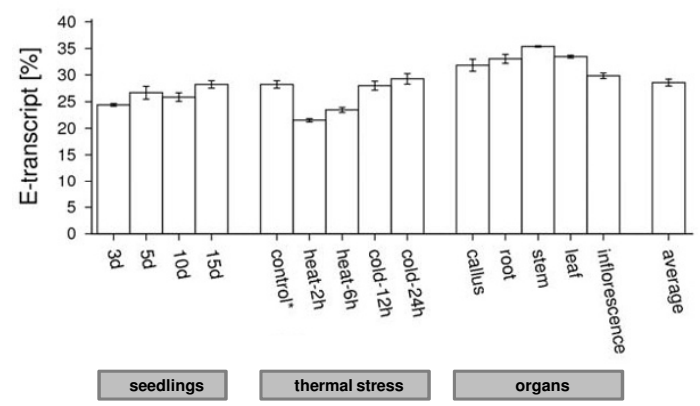

U2AF65

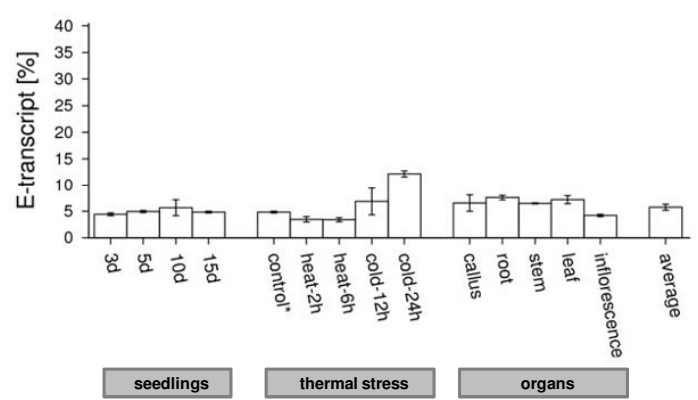

At3g54230

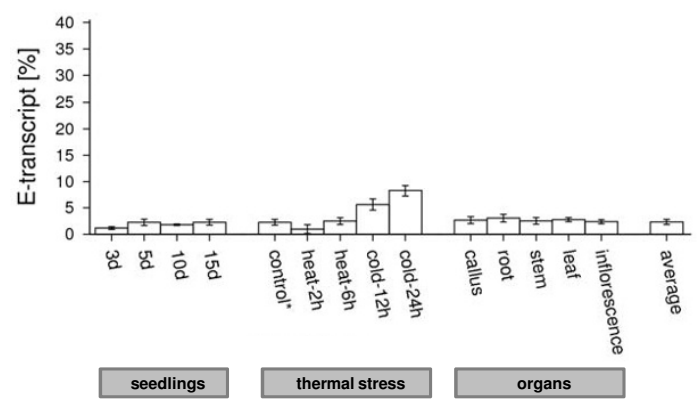

At5g59950

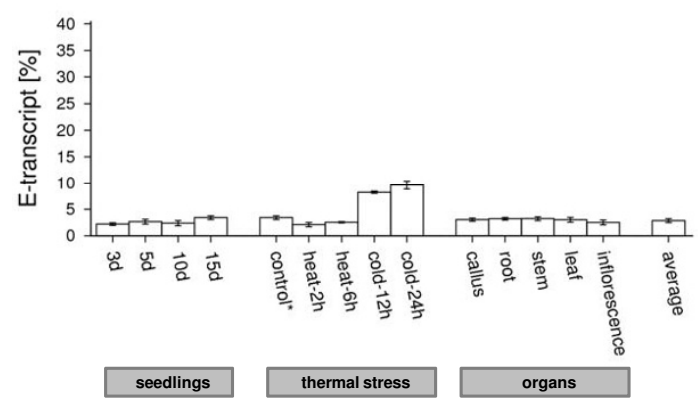

RS41

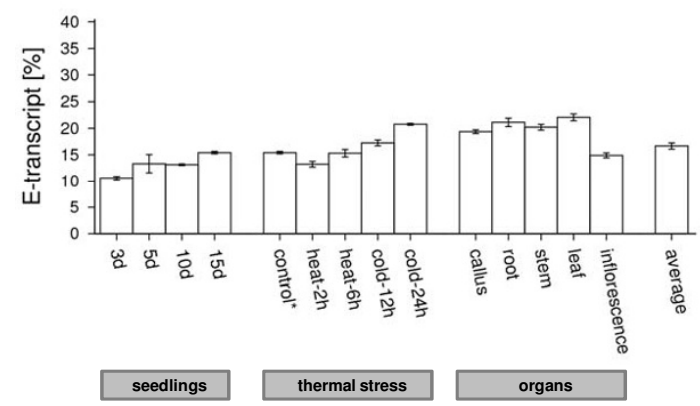

U11-35K

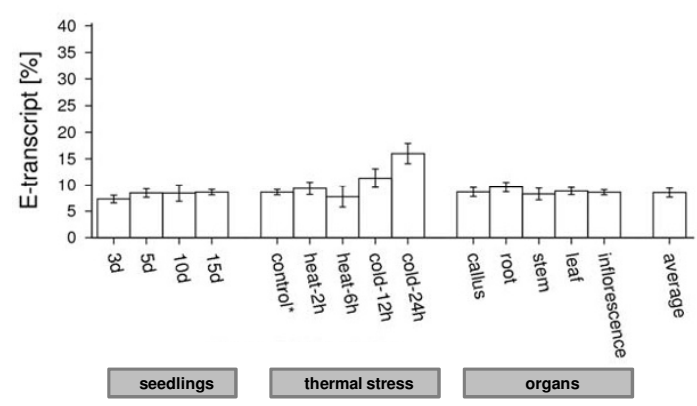

At2g24350

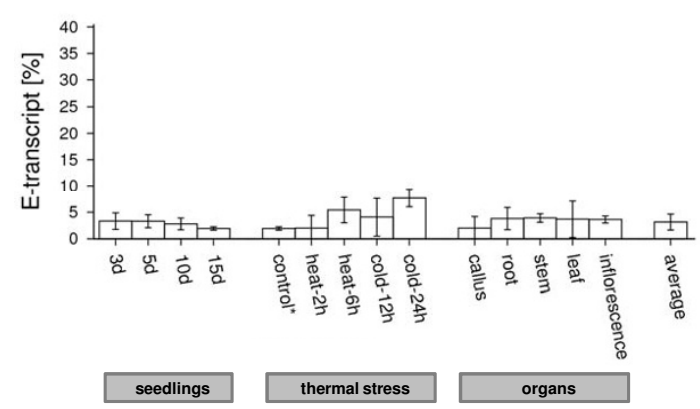

At3g54230

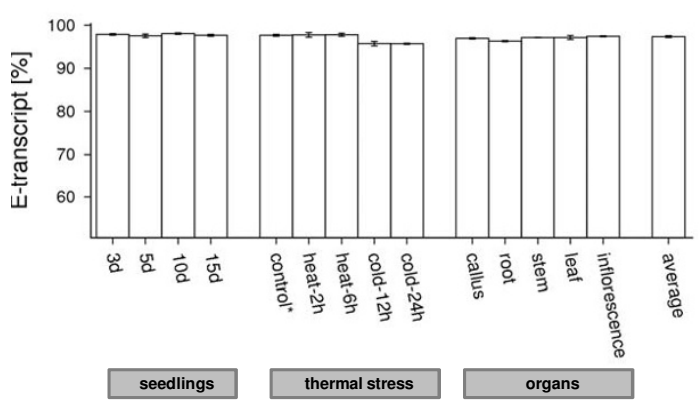

Figure I

E-transcript proportions in SR and SR-related protein-coding genes under several conditions. E-transcript proportions among different seedling ages, under heat and cold shock and in different organs are depicted. The I5d old seedlings also serve as the control for the thermal stress treatments, indicated by '*'. 
Table 3: E-transcript proportions in plant organs. The E-transcript frequencies in plant organs (root, leaf, stem, inflorescence) and callus are illustrated. Values were obtained from three independent experiments. Column 'ANOVA' displays the $p$-values, ' + ' $p \leq 0.05$ and ' $++\boldsymbol{+} \mathbf{p} \leq \mathbf{0 . 0}$ l.

\begin{tabular}{|c|c|c|c|c|c|c|}
\hline \multirow[b]{2}{*}{ Gene } & \multicolumn{6}{|c|}{ E-transcript(\%) } \\
\hline & Callus & Root & Stem & Leaf & Inflor & ANOVA \\
\hline RS4I & $19.4 \pm 0.4$ & $21.1 \pm 0.8$ & $20.2 \pm 0.6$ & $22.0 \pm 0.7$ & $14.9 \pm 0.5$ & ++ \\
\hline SR33/SCL33 & $31.8 \pm 1.2$ & $33.1 \pm 0.9$ & $35.4 \pm 0.2$ & $33.5 \pm 0.3$ & $29.8 \pm 0.6$ & ++ \\
\hline At2g24350 & $2.0 \pm 2.2$ & $3.8 \pm 2.1$ & $3.9 \pm 0.8$ & $3.7 \pm 3.5$ & $3.6 \pm 0.7$ & \\
\hline At3g54230 & $2.7 \pm 0.7$ & $3.0 \pm 0.8$ & $2.5 \pm 0.7$ & $2.8 \pm 0.4$ & $2.4 \pm 0.4$ & \\
\hline At4g35785 & $97.0 \pm 0.2$ & $96.3 \pm 0.2$ & $97.2 \pm 0.1$ & $97.2 \pm 0.5$ & $97.5 \pm 0.2$ & + \\
\hline At5g59950 & $3.0 \pm 0.3$ & $3.2 \pm 0.3$ & $3.2 \pm 0.4$ & $3.0 \pm 0.5$ & $2.5 \pm 0.5$ & \\
\hline UII-35K & $8.7 \pm 0.9$ & $9.6 \pm 1.0$ & $8.3 \pm 1.2$ & $8.8 \pm 0.7$ & $8.6 \pm 0.5$ & \\
\hline U2AF65 & $6.5 \pm 1.6$ & $7.6 \pm 0.5$ & $6.5 \pm 0.1$ & $7.2 \pm 0.8$ & $4.2 \pm 0.3$ & + \\
\hline
\end{tabular}

able to confer a spatial flexibility to the architecture of the spliceosome and thus may influence the splicing process and its outcome. Subtle changes in the protein composition induced by alternative splicing at NAGNAG acceptors could contribute to this flexibility as previously suggested [36]. Here we explored the degree of alternative splicing at NAGNAG acceptors in Arabidopsis in general and of SR and SR-related protein-coding genes in detail. In a genome-wide in silico screening we identified 6,772 introns that exhibit a NAGNAG acceptor motif. Out of this group, we identified 36 introns within 30 SR and SRrelated protein-coding genes. Intriguingly, NAGNAG acceptor motifs are more frequent in Arabidopsis SR and SR-related protein-coding genes $(36 \%)$ than on average $(21 \%)$. This is equivalent to the situation in human [36].

EST and mRNA data are the main resources to identify and locate alternative splicing of a gene. The total number of ESTs for a respective gene correlates with the diagnostic power of ESTs. Due to the relatively low EST coverage of the Arabidopsis genome, the EST data alone is not sufficient for a comprehensive characterization of the alternative splicing of NAGNAG motifs. For example, guided by the sequence-based prediction we could experimentally show an E-transcript frequency of 30\% for SR33/SCL33 despite initially lacking EST evidence for alternative splicing. This illustrates that the limitations of a low EST coverage can be at least in part circumvented with an appropriate prediction method. Currently, the EST data provides evidence for alternative splicing of 5\% (242 cases) of NAGNAG acceptors, which represents the lower bound for genome-wide estimates. On the other hand, our sequence-based prediction method suggested 33\% of genes produce minor isoforms with at least $2 \%$ frequency. But these predictions were found to be too optimistic, with only $53 \%$ of the cases actually giving detectable amounts of NAGNAG isoforms. Extrapolating these results to genome scale, $17 \%$ of Arabidopsis NAGNAGs are likely to be alternatively spliced. All prediction work neglects possible differences between tissues or developmental stages. In fact, our results for SR and SR-related protein genes indicate that organ- and development-specific, as well as stress-induced differences exist. However, the mechanisms that underlie tissue-specific regulation of alternative splicing are not yet understood and are not predictable by any current method.

Table 4: E-transcript proportions among different developmental stages. The E-transcript frequencies in seedlings of different ages (3d, 5d, 10d, 15d) are presented. Values were obtained from three independent experiments. Column 'ANOVA' displays the p-values, '+' $\mathrm{p} \leq \mathbf{0 . 0 5}$ and '++' $\mathrm{p} \leq \mathbf{0 . 0 1}$.

\begin{tabular}{|c|c|c|c|c|c|}
\hline \multirow[b]{2}{*}{ Gene } & \multicolumn{5}{|c|}{ E-transcript(\%) } \\
\hline & $3 d$ & $5 d$ & $10 d$ & $15 d$ & ANOVA \\
\hline RS4I & $10.6 \pm 0.4$ & $13.3 \pm 1.8$ & $13.1 \pm 0.2$ & $15.4 \pm 0.3$ & + \\
\hline SR33/SCL33 & $24.4 \pm 0.3$ & $26.7 \pm 1.3$ & $25.9 \pm 0.8$ & $28.2 \pm 0.7$ & + \\
\hline At2g24350 & $3.3 \pm 1.6$ & $3.3 \pm 1.3$ & $2.8 \pm 1.1$ & $1.9 \pm 0.4$ & \\
\hline At3g54230 & $1.2 \pm 0.3$ & $2.2 \pm 0.6$ & $1.8 \pm 0.2$ & $2.3 \pm 0.6$ & + \\
\hline At4g35785 & $97.9 \pm 0.2$ & $97.6 \pm 0.5$ & $98.1 \pm 0.2$ & $97.7 \pm 0.3$ & \\
\hline At5g59950 & $2.2 \pm 0.3$ & $2.6 \pm 0.5$ & $2.4 \pm 0.5$ & $3.4 \pm 0.4$ & + \\
\hline UII-35K & $7.3 \pm 0.8$ & $8.5 \pm 0.8$ & $8.4 \pm 1.6$ & $8.6 \pm 0.6$ & \\
\hline U2AF65 & $4.4 \pm 0.3$ & $4.9 \pm 0.3$ & $5.7 \pm 1.6$ & $4.8 \pm 0.2$ & \\
\hline
\end{tabular}


Table 5: E-transcript proportions under heat and cold shock. The E-transcript frequencies of seedlings kept in hot and cold conditions ( $2 \mathrm{~h}$ vs. $6 \mathrm{~h}$ and $12 \mathrm{~h}$ vs. $24 \mathrm{~h}$, respectively) compared to untreated seedlings. Values were obtained from three independent experiments. Column 'ANOVA' displays the $p$-values, ' + ' $p \leq 0.05$ and ' ++ ' $p \leq 0.01$.

\begin{tabular}{|c|c|c|c|c|c|c|c|}
\hline \multirow[b]{2}{*}{ Gene } & \multicolumn{5}{|c|}{ E-transcript(\%) } & \multicolumn{2}{|c|}{ ANOVA } \\
\hline & Untreated control & Heat shock $2 \mathrm{~h}$ & Heat shock $6 \mathrm{~h}$ & Cold shock 12 h & Cold shock 24 h & Heat shock & Cold shock \\
\hline RS4I & $15.4 \pm 0.3$ & $13.2 \pm 0.6$ & $15.3 \pm 0.8$ & $17.3 \pm 0.6$ & $20.7 \pm 0.2$ & + & ++ \\
\hline SR33/SCL33 & $28.2 \pm 0.7$ & $21.5 \pm 0.4$ & $23.5 \pm 0.5$ & $28.0 \pm 0.9$ & $29.3 \pm 1.0$ & ++ & \\
\hline At2g24350 & $1.9 \pm 0.4$ & $2.0 \pm 2.4$ & $5.4 \pm 2.5$ & $4.1 \pm 3.6$ & $7.7 \pm 1.7$ & & \\
\hline At3g54230 & $2.3 \pm 0.6$ & $1.0 \pm 0.9$ & $2.5 \pm 0.7$ & $5.6 \pm 1.1$ & $8.2 \pm 1.1$ & & + \\
\hline At4g35785 & $97.7 \pm 0.3$ & $97.8 \pm 0.6$ & $97.8 \pm 0.4$ & $95.8 \pm 0.6$ & $95.7 \pm 0.2$ & & + \\
\hline At5g59950 & $3.4 \pm 0.4$ & $2.1 \pm 0.5$ & $2.5 \pm 0.2$ & $8.2 \pm 0.3$ & $9.6 \pm 0.8$ & + & ++ \\
\hline UII-35K & $8.6 \pm 0.6$ & $9.4 \pm 1.2$ & $7.8 \pm 2.0$ & $11.3 \pm 1.8$ & $16.0 \pm 2.0$ & & + \\
\hline U2AF65 & $4.8 \pm 0.2$ & $3.5 \pm 0.6$ & $3.4 \pm 0.4$ & $6.9 \pm 2.6$ & $12.1 \pm 0.6$ & + & + \\
\hline
\end{tabular}

We found a negative correlation of the occurrence of alternatively spliced NAGNAG acceptors with the transcript levels of the genes. Though this finding needs further validation, it suggests that genes with high transcript abundance are not representative for the transcriptome. This would have profound consequences for studies extrapolating from highly expressed genes to the remaining transcriptome.

The effects of splicing factors are often dependent on their concentration, localization and phosphorylation, resulting in gradual changes of the alternative splicing pattern of certain transcripts $[2,16]$. Thus, splicing ratio changes, leading to differential abundance of splicing factor isoforms, could enhance the flexibility of the spliceosome composition and the splicing process itself. Hence, we asked whether this is the case for the genes shown to have an alternatively spliced NAGNAG. We experimentally tested several organs, developmental stages and environmental influences. Interestingly, significant organ-specific differences of splicing ratios were detected in four cases. Most notably, inflorescence showed reduced splicing of the minor acceptor in nearly all experimental candidates. A very similar effect was seen in early developmental stages ( $3 \mathrm{~d}$ compared to later stages). A common reason may be that stem cells, enriched in both these samples, disfavor minor acceptor usage. This should be further tested in future experiments. Finally, the most pronounced effect on the splicing ratio was seen after cold shock, consistent with previous observations $[21,47]$.

For the analyzed gene family, it seemed reasonable to ask for the impact of NAGNAG splicing on the RRM domain. We found that none of the eight NAGNAG acceptors in SR proteins do affect the RRM. In contrast, 12 of the 23 SRrelated proteins have a NAGNAG acceptor located in the RRM domain. Previously, functional differences due to a NAGNAG acceptor in the RRM were observed for the U11-35K protein [42] and, more generally, NAGNAG alternative splicing in RRM-containing proteins was suggested to have an impact on the tertiary structures [41]. Also the usage of the E-acceptor site results in a protein with one additional serine in SR33/SCL33 and RS41. Serine residues in SR proteins are the targets of phosphorylation, and numerous studies have shown that the phosphorylation status of SR proteins is critical for their splicing activity as well as subcellular localization $[2,14,16,27]$.

Most notably, the pattern of differential splicing ratios is similar for all analyzed genes. Thus, the differential effects on NAGNAG alternative splicing seem to be organ- and condition-specific rather than gene-specific. This favors the hypothesis that differential splicing of NAGNAG acceptors is mostly independent of sequence-specific splicing regulators, and is rather mediated by (subtle) organ- and condition-specific differences of the spliceosomal core composition. Intuitively, such lack of tight regulation seems to argue against a functional relevance of splice variants, as was suggested earlier [44]. However, several tandem splice sites with clear functional implications exhibit constant splicing ratios. Vice versa, it was shown that alternative splicing events producing variable splicing ratios do not always imply a function [48]. Surely, the functional relevance of the alternative splice events analyzed in this study remains to be evaluated.

\section{Conclusion}

We demonstrated, that NAGNAG acceptors frequently occur in the Arabidopsis genome and are particularly prevalent in SR and SR-related protein-coding genes. Insufficient EST coverage can be compensated using the sequence-based method to predict alternative splicing of NAGNAG acceptors. The observed differential effects on NAGNAG alternative splicing appear to be organ- and condition-specific rather than gene-specific. In particular, inflorescence and early seedling stages consistently show reduced levels of the minor transcript isoforms. 


\section{Methods}

\section{Screening for NAGNAG acceptor tandems}

The annotated genome sequence of Arabidopsis thaliana was obtained from GenBank, to serve as a data basis for the locations of intron-exon boundaries and their sequence. Boundaries with the sequence NAGNAG| or NAG|NAG (where "|" indicates the annotated boundary) were sampled. Redundancies due to annotation of multiple transcript isoforms were filtered. Potential splice variants derived from the genomic NAGNAG patterns were detected and quantified by a WU-BLASTN search of 60-nt sequence windows around the resulting exon-exon junctions against all Arabidopsis ESTs from TIGR and RIKEN databases [49,50], using parameters $\mathrm{W}=13 \mathrm{~N}=-8$ nogap $\mathrm{S}=180 \mathrm{hspmax}=1$. BLAST matches were considered valid if perfect sequence identity was found in a 12-nt window around the exon-exon junctions [51].

\section{Prediction of splicing ratios}

All NAGNAG-containing introns with supporting EST data for E- and I-acceptor were divided into subsets of 64 motif classes, according to the heptameric motif NAGNAGN. Maximum-likelihood estimators for E-to-I transcript ratios were calculated by combining the EST counts per class. In order to prevent a bias caused by cases with an extremely high EST coverage, counts were limited to a maximum of 10 per isoform per NAGNAG site, and eventually downscaled.

\section{Identification of SR/SR-related protein genes}

The complete set of non-redundant Arabidopsis proteins was screened for existing RNA-recognition motifs, and its derivatives, using Pfam HMM definitions (PF00076, PF04059, PF08777) and hmmsearch (HMMer package, [52]) applying recommended cutoff parameters. Additionally, the relative content of RS or SR dipeptides of each gene product was determined. A significance threshold $>0.016$ for $\mathrm{R} / \mathrm{S}$-richness was applied, corresponding to the transition point of a two-exponential case distribution. A subset of 84 proteins had both significant RRM profile hits and R/S-rich sequence. Of these 84, 19 are identified as SR proteins sensu strictu $[18,19]$.

\section{Plant material and stress treatments}

A. thaliana ecotype Columbia seedlings were grown on Murashige and Skoog (MS) medium at $22^{\circ} \mathrm{C}$ with $16 \mathrm{~h} / 8$ $\mathrm{h}$ light/dark cycle and harvested after $3 \mathrm{~d}, 5 \mathrm{~d}, 10 \mathrm{~d}$ and $15 \mathrm{~d}$. Callus tissue was generated from roots of one-week-old seedlings by transferring them onto a callus induction medium ( $1 \times$ Gamborg's B5 medium, 2\% glucose, $0.5 \mathrm{~g} / \mathrm{l}$ MES (pH 5.7), 0.8\% agar, $0.5 \mathrm{mg} / \mathrm{l}$ 2,4-D [2,4-Dichlorphenoxyacetate] and $0.005 \mathrm{mg} / \mathrm{l}$ kinetin). Callus tissue was collected and frozen in liquid nitrogen for RNA extraction. Heat and cold stress treatments were done with $15 \mathrm{~d}$-old seedlings. Seedlings were grown for 15 -days and exposed to heat $\left(38^{\circ} \mathrm{C}\right)$ for two and six hours or cold $\left(4^{\circ} \mathrm{C}\right)$ for 12 and 24 hours, the untreated control seedlings were kept at $22^{\circ} \mathrm{C}$ for the corresponding time period.

\section{RT-PCR and splice-variant analysis by quantitative capillary electrophoresis}

RNA from plant tissues, seedlings or callus was isolated using RNeasy Plant Mini Kit (Qiagen) and quantified spectrophotometrically at $260 \mathrm{~nm}$. RNA was treated with DNAseI and used to synthesize first strand cDNA with oligo (dT) primer using SuperScriptII (Invitrogen). For validation of splice variants, three independent RT-PCRs for each candidate were performed with cDNA from different organs, developmental seedling stages and stress treatments to yield amplicons covering the respective exon-exon junction. Reactions were set up with BioMix Red (Bioline, Randolph, USA) and 10 pmol primer in 50 $\mu \mathrm{l}$ total volume, according to the manufacturer's instructions. Each forward primer was labelled with 6-carboxyfluorescein (FAM) for subsequent analysis on a capillary sequencer. The thermocycle protocol was $1 \mathrm{~min} 30 \mathrm{sec}$ initial denaturation at $94^{\circ} \mathrm{C}$, followed by 35 cycles of $50 \mathrm{sec}$ denaturation at $94^{\circ} \mathrm{C}, 45 \mathrm{sec}$ annealing at $55-59^{\circ} \mathrm{C}, 1$ min extension at $72^{\circ} \mathrm{C}$, and a final $1 \mathrm{~h}$ extension step at $72^{\circ} \mathrm{C}$. The following gene-specific primers were used: RS41 reverse GCTGGCGGCGAACGAGA, RS41 forward GAGAAGGGAAAGCAGGAGTC, SR33 forward GCTGCTGATGCAAAACATC, SR33 reverse CTCCCATCATATCGCT CTTC, SRZ22a forward CGTGGTGGTTCTGATTTGAAG, $S R Z 22 a$ reverse GATCTAGCACGAGGGCTGTAA, SR45i9 forward GTCGCTCTCGTTCAAGTTCC, SR45i9 reverse TTTACGAGGTGGAGGTGGTG, SR45i7 forward AGGCCG TTCTCCATCTTCTC, SR45i7 reverse CCTTCTGGGACTTGGTGAAC, At2g24350 forward CTGCGCTCTGTCATTGTTTC, At2g24350 reverse ACATGAGGCTCCGTTTCTTG, At1g53650 forward AGTTCTTCGCTTTGCGTTTG, At1g53 650 reverse GCAGGCAGACTGAAAGAAGG, At5g09880 forward GGAAGAGAAGGAACCCGAAG, At5g09880 reverse CCATTGGAACTGACATCACG, At5g59950 forward TGGATGGAAAACCCATGAAG, At5g59950 reverse ACCACCTCGTTGTTGACCTC, At1g76940 forward ACATCATCCTCCTGGTGGTC, At1g76940 reverse CCACCTTCTCCTGATTGCAC, At2g43370 forward GGAGCTTCAC GAGGATATGG, At2g 43370 reverse CTCAGGCGGAAGCT GAATAC, At4g35785 forward ATCTCCTTCACCCCGAAA AG, At4g35785 reverse CAAGACGCAACCTTTCCTTC, At1g60900 forward GCGCCTCCTGATATGTTAGC, At1 1960 900 reverse AGGCCACCAACATAGACTCG, At3g54230 forward GGGTCCTTTGCATCATGTTC, At3g54230 reverse ACATCCGCTGAAGGAGAATC. The PCR products were appropriately diluted (1/20 to $1 / 50)$ and 1 ul was supplemented with $10 \mathrm{ul}$ formamide (Roth, Karlsruhe) and 0.5 ul of GeneScan 500 LIZ (Applied Biosystems). The mixture was than separated on an ABI 3730 capillary 
sequencer and analyzed with the GeneMapper 4.0 software. The E-transcript proportion (\%) was calculated as follows: peak area for the E-isoform/(E-isoform+I-isoform) $\times 100$.

\section{Splice-variant analysis by clone-counting}

For validation of splice variants (RS41, SR33, SRZ22a, SR45), RT-PCR was performed with cDNA from root, leaf, stem and inflorescence to yield amplicons covering the respective exon-exon junction. The following gene-specific primers were used: RS41 forward 5'-AAG AGG AGG GAA AGC AGGAG-3' and reverse 5'-GCG ATT TCG AAT GGA GTC AT-3'; SRZ22a forward 5'-GCA AGA ATG GAT GGA GGG TA-3' and reverse 5'-CCA CGA GGA GAA GGA CTA CG-3'; SR33/SCL33 forward 5'-AGG GTT TGG GTT CGT TCA AT-3' and reverse 5'-CTC CGT GAC CGA GAT CTA CC-3'; SR45 forward 5'-CAC CTC CAA GGA GAC TAC GC-3' and reverse 5'-CAG TGG CCT CTT AGG ACT GC-3'. PCR products were gel purified using the QIAquick Gel Extraction Kit and the isolated fragments were cloned into pCR2.1-TOPO (Invitrogen) according to the supplier's recommendations. 25 clones per gene and plant sample were selected and Sanger-sequenced using M13 standard reverse primer (20-mer). Sequence analysis was performed using SPIDEY [53].

\section{Authors' contributions}

SS performed the capillary electrophoresis experiments, analyzed and interpreted data and wrote the initial manuscript. KS conceived and designed experiments, performed the statistical analyses, interpreted data and contributed to the manuscript. $\mathrm{MH}$ performed the genome-wide screening. GSA and SGP performed experiments, analyzed and interpreted data. ASNR, RB and MP as principal investigators conceived the experiments. All authors contributed to the final manuscript.

\section{Additional material}

\section{Additional file 1}

All NAGNAG acceptor cases identified within the Arabidopsis genome. The absolute numbers of ESTs supporting the E- or the I-acceptor ("ESTs_E" and "ESTs_I", respectively) and the sequence-based prediction of E-transcript frequency ("expected_E") are given.

Click here for file

[http://www.biomedcentral.com/content/supplementary/14712164-9-159-S1.xls

\section{Additional file 2}

Counts of Arabidopsis NAGNAG cases depending on local EST coverage. The number of cases at least reaching a given coverage is presented. Click here for file

[http://www.biomedcentral.com/content/supplementary/14712164-9-159-S2.xls]

\section{Additional file 3}

Heptamer motif classification. Heptameric motif classes are presented with corresponding maximum-likelihood estimators for E-to-I-transcript ratios, used for sequence-based prediction.

Click here for file

[http://www.biomedcentral.com/content/supplementary/1471-

2164-9-159-S3.xls]

\section{Additional file 4}

Comprehensive list of experimental values from all analyzed SR and SR-related protein-coding genes. Column 'avg' lists the averaged E-transcript proportions derived from three independent experiments per probe with corresponding standard deviation in column 'sd'. The values derived from the $3 d, 5 d, 10 d, 15 d$ old seedlings, callus and organs were averaged (column 'avg organs-seedlings') as well as the corresponding standard deviations ('avg error') to gain appropriate values for a comparison with the sequence-based predictions (see column 'predicted'). Grey shaded values mark the cases where NAGNAG alternative splicing was validated $[($ avg error $\times 2)<($ avg organs-seedlings $)]$.

Click here for file

[http://www.biomedcentral.com/content/supplementary/14712164-9-159-S4.xls]

\section{Acknowledgements}

This work was supported by grants from the German Ministry of Education and Research (0313652D) and the Deutsche Forschungsgemeinschaft (SFB604-02) to M.P as well as the Department of Energy (DE-FG0204ERI5556) to A.S.N.R.

\section{References}

I. Lareau LF, Green RE, Bhatnagar RS, Brenner SE: The evolving roles of alternative splicing. Curr Opin Struct Biol 2004, I 4(3):273-282.

2. Reddy AS, N : Alternative splicing of pre-messenger RNAs in plants in the genomic era. Annu Rev Plant Biol 2007, 58:267-294.

3. Stamm S, Ben-Ari S, Rafalska I, Tang Y, Zhang Z, Toiber D, Thanaraj TA, Soreq H: Function of alternative splicing. Gene 2005, 344: $1-20$.

4. Cartegni L, Chew SL, Krainer AR: Listening to silence and understanding nonsense: exonic mutations that affect splicing. Nat Rev Genet 2002, 3(4):285-298.

5. Kim E, Magen A, Ast G: Different levels of alternative splicing among eukaryotes. Nucleic Acids Res 2007, 35(I): I25-I3I.

6. Campbell MA, Haas BJ, Hamilton JP, Mount SM, Buell CR: Comprehensive analysis of alternative splicing in rice and comparative analyses with Arabidopsis. BMC Genomics 2006, 7:327.

7. Wang BB, Brendel V: Genomewide comparative analysis of alternative splicing in plants. Proc Natl Acad Sci USA 2006, 103(18):7175-7|80.

8. Ner-Gaon H, Halachmi R, Savaldi-Goldstein S, Rubin E, Ophir R, Fluhr $R$ : Intron retention is a major phenomenon in alternative splicing in Arabidopsis. Plant J 2004, 39(6):877-885.

9. Lorkovic ZJ, Wieczorek Kirk DA, Lambermon MH, Filipowicz W: Pre-mRNA splicing in higher plants. Trends Plant Sci 2000, 5(4): $160-167$.

10. Haas BJ, Delcher AL, Mount SM, Wortman JR, Smith RK Jr, Hannick LI, Maiti R, Ronning CM, Rusch DB, Town CD, Salzberg SL, White O: Improving the Arabidopsis genome annotation using maximal transcript alignment assemblies. Nucleic Acids Res 2003, 3I(19):5654-5666.

II. Sakurai T, Satou M, Akiyama K, lida K, Seki M, Kuromori T, Ito T, Konagaya A, Toyoda T, Shinozaki K: RARGE: a large-scale database of RIKEN Arabidopsis resources ranging from transcriptome to phenome. Nucleic Acids Res 2005:D647-650.

12. Moskal WA Jr, Wu HC, Underwood BA, Wang W, Town CD, Xiao $Y$ : Experimental validation of novel genes predicted in the 
un-annotated regions of the Arabidopsis genome. BMC Genomics 2007, 8: 18 .

13. Black DL: Mechanisms of alternative pre-messenger RNA splicing. Annu Rev Biochem 2003, 72:291-336.

14. Manley JL, Tacke R: SR proteins and splicing control. Genes Dev 1996, I0:1569-1579.

15. Fu XD: The superfamily of arginine/serine-rich splicing factors. RNA 1995, I(7):663-680.

16. Graveley BR: Sorting out the complexity of SR protein functions. RNA 2000, 6(9):||

17. Wang BB, Brendel V: The ASRG database: identification and survey of Arabidopsis thaliana genes involved in pre-mRNA splicing. Genome Biol 2004, 5( I 2): R I02.

18. Lorkovic Z], Barta A: Genome analysis: RNA recognition motif (RRM) and K homology (KH) domain RNA-binding proteins from the flowering plant Arabidopsis thaliana. Nucleic Acids Res 2002, 30(3):623-635.

19. Reddy AS: Plant serine/arginine-rich proteins and their role in pre-mRNA splicing. Trends Plant Sci 2004, 9( I I):54I-547.

20. Kalyna $M$, Barta $A:$ A plethora of plant serine/arginine-rich proteins: redundancy or evolution of novel gene functions? Biochem Soc Trans 2004, 32(Pt 4):56I-564.

21. Palusa SG, Ali GS, Redddy ASN: Alternative splicing of premRNAs of Arabidopsis serine/arginine-rich proteins and its regulation by hormones and stresses. Plant J 2007, 49:1091-II07.

22. Kalyna M, Lopato S, Voronin V, Barta A: Evolutionary conservation and regulation of particular alternative splicing events in plant SR proteins. Nucleic Acids Res 2006, 34( I 6):4395-4405.

23. Golovkin M, Reddy AS: An SC35-like protein and a novel serinel arginine-rich protein interact with Arabidopsis UI-70K protein. I Biol Chem 1999, 274(5 I):36428-36438.

24. Lazar G, Schaal T, Maniatis T, Goodman HM: Identification of a plant serine-arginine-rich protein similar to the mammalian splicing factor SF2/ASF. Proc Natl Acad Sci USA 1995, 92(17):7672-7676.

25. Lopato S, Kalyna M, Dorner S, Kobayashi R, Krainer AR, Barta A atSRp30, one of two SF2/ASF-like proteins from Arabidopsis thaliana, regulates splicing of specific plant genes. Genes Dev 1999, 13(8):987-1001.

26. Lopato S, Waigmann E, Barta A: Characterization of a novel arginine/serine-rich splicing factor in Arabidopsis. Plant Cell 1 996, 8( I 2):2255-2264

27. Ali GS, Reddy AS: ATP, phosphorylation and transcription regulate the mobility of plant splicing factors. J Cell Sci 2006 , I I 9(Pt | 7):3527-3538

28. Isshiki $M$, Tsumoto $A$, Shimamoto $K$ : The serine/arginine-rich protein family in rice plays important roles in constitutive and alternative splicing of pre-mRNA. Plant Cell 2006, I8(I): I 46-158.

29. Kalyna M, Lopato S, Barta A: Ectopic expression of atRSZ33 reveals its function in splicing and causes pleiotropic changes in development. Mol Biol Cell 2003, I4(9):3565-3577.

30. Lazar G, Goodman HM: The Arabidopsis splicing factor SRI is regulated by alternative splicing. Plant Mol Biol 2000, 42(4):57I-58I.

31. Luehrsen KR, Taha S, Walbot V: Nuclear pre-mRNA processing in higher plants. Prog Nucleic Acid Res Mol Biol 1994, 47: I 49-193.

32. Reddy AS, N : Nuclear Pre-mRNA Splicing in Plants. Crit Rev, Plant Sci 200I, 20:523-57I.

33. Simpson GG, Filipowicz W: Splicing of precursors to mRNA in higher plants: mechanism, regulation and sub-nuclear organisation of the spliceosomal machinery. Plant Mol Biol 1996, 32(I-2): I-4I.

34. Dou Y, Fox-Walsh KL, Baldi PF, Hertel KJ: Genomic splice-site analysis reveals frequent alternative splicing close to the dominant splice site. RNA 2006, I 2(I 2):2047-2056.

35. Akerman $M$, Mandel-Gutfreund $Y$ : Alternative splicing regulation at tandem 3' splice sites. Nucleic Acids Res 2006, 34(I):23-3I.

36. Hiller M, Huse K, Szafranski K, Jahn N, Hampe J, Schreiber S, Backofen $R$, Platzer M: Widespread occurrence of alternative splicing at NAGNAG acceptors contributes to proteome plasticity. Nat Genet 2004, 36(I2): 1255-1257.

37. Ferranti $P$, Lilla S, Chianese L, Addeo F: Alternative nonallelic deletion is constitutive of ruminant alpha(sI)-casein. J Protein Chem 1999, 18(5):595-602.
38. Rogina B, Upholt WB: The chicken homeobox gene Hoxd-I I encodes two alternatively spliced RNA species. Biochem Mol Biol Int 1995, 35(4):825-831.

39. Li L, Howe GA: Alternative splicing of prosystemin pre-mRNA produces two isoforms that are active as signals in the wound response pathway. Plant Mol Biol 200I, 46(4):409-4I9.

40. Hiller M, Szafranski K, Sinha R, Huse K, Nikolajewa S, Rosenstiel P, Schreiber S, Backofen R, Platzer M: Assessing the fraction of short-distance tandem splice sites under purifying selection. RNA 2008 in press.

4I. lida K, Shionyu M, Suso Y: Alternative splicing at NAGNAG acceptor sites shares common properties in land plants and mammals. Mol Biol Evol 2008, 25(4):709-7I8.

42. Lorkovic Z], Lehner R, Forstner C, Barta A: Evolutionary conservation of minor $U$ I 2-type spliceosome between plants and humans. RNA 2005, I I(7): I095-II07.

43. Lareau LF, Inada M, Green RE, Wengrod JC, Brenner SE: Unproductive splicing of SR genes associated with highly conserved and ultraconserved DNA elements. Nature 2007, 446(7| 38):926-929.

44. Chern TM, van Nimwegen E, Kai C, Kawai J, Carninci P, Hayashizaki $\mathrm{Y}$, Zavolan M: A simple physical model predicts small exon length variations. PLoS Genet 2006, 2(4):e45.

45. Tsai KW, Tarn WY, Lin WC: Wobble splicing reveals the role of the branch point sequence-to-NAGNAG region in 3' tandem splice site selection. Mol Cell Biol 2007, 27( 16):5835-5848.

46. Birney E, Kumar S, Krainer AR: Analysis of the RNA-recognition motif and RS and RGG domains: conservation in metazoan pre-mRNA splicing factors. Nucleic Acids Res 1993, 2I(25):5803-58I6.

47. Fung RW, Wang CY, Smith DL, Gross KC, Tao Y, Tian M: Characterization of alternative oxidase (AOX) gene expression in response to methyl salicylate and methyl jasmonate pretreatment and low temperature in tomatoes. I Plant Physiol 2006, 163(10):1049-1060

48. Hiller M, Platzer M: Widespread and subtle: alternative splicing at short-distance tandem sites. Trends Genet 2008 in press.

49. RIKEN Arabidopsis Full-Length Clone Databse [ftp://pfg web.gsc.riken.jp/rafl/sequence/]

50. The TIGR Arabidopsis thaliana Database [http://www.tigr.org/ tigr-scripts/tgi/T index.cgi?species=arab]

51. Thanaraj TA: A clean data set of EST-confirmed splice sites from Homo sapiens and standards for clean-up procedures. Nucleic Acids Res 1999, 27( (3):2627-2637.

52. Eddy SR: Profile hidden Markov models. Bioinformatics 1998 , I 4(9):755-763

53. Wheelan SJ, Church DM, Ostell JM: Spidey: a tool for mRNA-togenomic alignments. Genome Res 200I, I I (I I): 1952-1957.
Publish with Bio Med Central and every scientist can read your work free of charge

"BioMed Central will be the most significant development for disseminating the results of biomedical research in our lifetime. "

Sir Paul Nurse, Cancer Research UK

Your research papers will be:

- available free of charge to the entire biomedical community

- peer reviewed and published immediately upon acceptance

- cited in PubMed and archived on PubMed Central

- yours - you keep the copyright

Submit your manuscript here:

http://www.biomedcentral.com/info/publishing_adv.asp
BioMedcentral 